\title{
Tumor microenvironment: bidirectional interactions between cancer cells and normal cells
}

\author{
Lu-Yuan Li ${ }^{\bowtie}$ \\ College of Pharmacy, Nankai University, Tianjin 300071, China \\ $\triangle$ Correspondence: liluyuan@nankai.edu.cn
}

\begin{abstract}
"The road to metastasis is paved with tumor-microenvironment interactions", claimed Dr. Isaac Witz from Tel Aviv University, Israel, in his keynote speech at the first Tianjin Forum on Tumor Microenvironment (http://TFTM.nankai.edu. $\mathrm{cn}$ ), an international conference held at Nankai University in Tianjin, China, on July 2-4, 2010. About 300 cancer researchers and students attended the conference. Thirty lecturers from North America, Europe and Asia discussed their findings on how the interactions between cancer cells and host normal cells in the microenvironment may determine whether the cancer cells develop into a malignant tumor, metastasize, or remain dormant, even die.

Dr. Witz briefly reviewed in a historic perspective how cancer researchers came to realize the importance of the interactions between cancer cells and their microenvironment in the processes of cancer development and metastasis. He pointed out that there are two types of microenvironments that cancer cells reside in: the microenvironment of the primary, local tumor and the microenvironment of secondary, metastatic sites. He emphasized that the interactions between tumor cells and their microenvironment are dynamic and bi-directional and that each interaction partner shapes the phenotype of the other partner. The relationship between cancer cells and their microenvironment is often likened to "seed and soil". The hypothesis was first put forward by Stephen Paget (Lancet 1:571, 1889). "What is it that decides what organs shall suffer a case of disseminated cancer?" Paget wondered. "When a plant goes to seed, its seeds are carried in all directions," he wrote. "But they can only live and grow if they fall on congenial soil." It may be useful to point out the conceptual differences between Paget's perception and the modern paradigm. According to Paget's hypothesis the microenvironment either supports metastasis by supplying growth promoting factors or alternatively inhibits metastasis by growth inhibitors. The
\end{abstract}

contemporary paradigm, however, assigns to the tumor microenvironment an inductive, adaptive and selective function: The tumor is directed into one or several possible molecular evolution pathways by signals originating in native or modified microenvironmental factors. Many of these pathways may lead to metastasis.

Dr. Robert Kerbel from University of Toronto, another keynote speaker, discussed circumstances by which certain cytotoxic drugs can rapidly alter the tumor microenvironment in such a way as to decrease their efficacy. Specifically, he summarized results showing that bolus injections of certain cytotoxic chemotherapy agents at maximum tolerated doses such as paclitaxel or cyclophosphamide, or microtubule inhibiting 'vascular disrupting agents' can cause a rapid mobilization of various cell populations from the bone marrow compartment, which then migrate to and colonize the drug treated tumors. These retained cell populations include endothelial progenitor cells, but likely other cell types as well including monocytes, macrophages, and mesenchymal derived stem cells. These cell populations can promote tumor repopulation, at least in part, by stimulating tumor angiogenesis and vasculogenesis. This acute reactive host response appears to be driven by systemic induction of multiple chemokines and cytokines such as granulocyte colonystimulating factor (G-CSF) and stromal derived factor-1 (SDF-1). The mobilization/tumor colonization response by such bone marrow-derived cells (BMDC) can be blocked, at least in part, by several pharmacologic maneuvers. These include administration of vascular endothelial cell growth factor (VEGF) pathway targeting antibodies (which, incidentally, provides potential explanation about how administration of drugs such as bevacizumab may enhance the efficacy of chemotherapy, i.e., by suppressing such host BMDC responses and thus extending the duration of the tumor response), or by anti-SDF-1 antibodies. 
In addition, Dr. Kerbel discussed the concept of metronomic chemotherapy-frequent treatment with low dosages. Indeed, metronomic chemotherapy not only avoids the rapid chemokine/cytokine/BMDC response, but can actually target certain BMDCs such as endothelial progenitor cells, and perhaps others as well. As such, this helps explain why lower doses of chemotherapy drug administered in a frequent, regular fashion with no breaks, despite being unable to induce rapid tumor regressions, might still possess anti-tumor activity that is equal or superior to conventional maximum tolerated doses chemotherapy regimens. Dr. Kerbel went on to summarize results employing new preclinical models of advanced metastatic disease showing remarkable antitumor effects of certain metronomic chemotherapy regimens used either alone or in combination with a targeted antiangiogenic VEGF pathway targeting drug. Some of these preclinical results have now led to the initiation and completion of phase II clinical trials evaluating similar therapeutic regimens in patients with advanced disease, with highly encouraging results, both in terms of efficacy and reduced toxicity, and as such, are leading to phase III clinical trial evaluation.

The meeting was organized into several sessions to address various aspects of the tumor microenvironment. One included discussions on tumor fluids, vasculature, and lymphatic. Dr. Bruce Zetter from Harvard Medical School spoke about the fluid microenvironment in prostate cancer. Prostate cancer cells grow slowly in the prostate but quickly in the bone. Spermine-a polyamine that in the prostate is 100 times more than elsewhere-is responsible for the inhibition. Ornithine decarboxylase is upregulated in the cell cycle in $\mathrm{G} 1$. It converts ornithine to putrescine, then to Spermidine, then to spermine. The ductal epithelial cells surrounded by the fluid still differentiate early. With prostate cancer progression, the prostate cancer cells either move away from the spermine, or the cells dedifferentiate and stop making spermine, or become resistant to spermine. All three things happen.

Dr. Lu-Yuan Li from Nankai University spoke about modulation of bone marrow derived hematopoietic stem cell (HSC) differentiation into endothelial progenitor cells (EPC) by a tumor necrosis factor (TNF) family member, vascular endothelial cell growth inhibitor (VEGI; TNFSF15). VEGI is produced by endothelial cells, induces apoptosis in proliferating endothelial cells, and is down-regulated in tumor vasculature. Using a cell culture model to study differentiation of mouse bone marrow-derived HSC to EPC, a process accompanied by decreasing stem cell markers and increasing endothelial cell markers, he showed that VEGI inhibits EPC adhesion on fibronectin, laminin, and vitronectin, as well as EPC migration and capillary formation in vitro. Additionally, VEGI induces apoptosis to E-selectin positive adhered EPC but exerts a protective effect on EPC in suspension. Death receptor-3 appears to be partially responsible for VEGIinduced cell death in EPC.
Dr. Zhou Wang from University of Pittsburgh, USA, spoke on regulation of angiogenesis by tumor suppressor U19/Eaf2 (ELL-associated factor 2), an androgen responsive gene in the prostate. Dr. Shi-Yuan Cheng, also from University of Pittsburgh, discussed mechanisms of platelet-derived growth factor receptor- $\alpha$ (PDGFRa)-promoted glioma invasion and inhibition of growth and angiogenesis of brain gliomas. Dr. Yongzhang Luo of Tsinghua University, China, spoke on the effect of pulmonary vascular destabilization in the premetastatic phase on lung metastasis. He showed that, in the premetastatic stage, angiopoietin 2 (Ang2), matrix metalloproteinase-3 (MMP-3) and MMP-10 are upregulated in the lung by primary B16/F10 tumor, which leads to the increased permeability of pulmonary vasculatures and extravasation of circulating tumor cells. Ang2, MMP3, and MMP10 have a synergistic effect on disrupting vascular integrity in both in vitro and in vivo models. He concluded that pulmonary vascular destabilization in the premetastatic phase promotes the extravasation of tumor cells and facilitates lung metastasis, which may provide potential targets for clinical prevention of metastasis. Dr. Tuomas Tammela from University of Helsinki, Finland, spoke about the importance of lymphangiogenesis in pathological conditions such as ischemic heart disease and critical lower limb ischemia. He pointed out that intralymphatic tumor cells in metastatic transit inside lymphatic vessels serve as putative foci of tumor relapse in murine tumor models as well as in human melanoma.

The bone microenvironment was another interesting topic at the meeting. Dr. Evan Keller from University of Michigan Medical Center, USA, discussed the biology of prostate cancer bone metastases. Prostate metastasizes to bone, liver, lymph node, lung, soft tissue, dura, adrenal in that order. Bone resorption enhances carcinoma migration. Stromalderived factor (SDF-1) and its receptor CXCR4 play a major role. Prostate cancer bone metastasis is heterogeneous. It favors the vertebrae, ribs, skull and proximal long bones, forming on trabecular bone surfaces at sites of previous bone resorption, coinciding with increased serum markers of resorption, and causing high morphometric resorption of bone. Osteoprotegerin inhibits this. Phase III study of denosumab, an antibody to RANKL, in men with hormonerefractory prostate cancer is underway. Signaling pathways involving BMP and Wnt were discussed.

Dr. David Roodman from University of Pittsburgh School of Medicine spoke on osteoblast suppression in myeloma. Multiple myeloma patients frequently have bone lesions. Dr. Roodman discussed the role of soluble factors produced by myeloma cells, including TNFa and interleukin-7, which suppress osteoblast differentiation, and do so by upregulating the transcriptional repressor Gfi-1. Experimental evidence from his lab suggests that Gfi- 1 is an important transcriptional suppressor of Runx 2 in osteoblast precursors in myeloma. Dr. Cun-Yu Wang from University of California, Los Angels, spoke about molecular regulation of breast cancer bone 
metastasis by transcription factor NFkB. Dr. Xu Cao from Johns Hopkins University, USA, spoke about transforming growth factor- $\beta 1$ (TGF $\beta 1$ )-induced migration of bone marrow stem cells to couple bone resorption and formation and implication for treatment of bone diseases including metastasis of tumors to the skeleton.

Dr. Guozhi Xiao, also from University of Pittsburgh, discussed about activating transcription factor-4 (ATF4) regulation of osteoclast differentiation and its implication in skeletal metastases. He demonstrated that both in vitro and in vivo osteoclast differentiation is severely impaired by lack of ATF4 in a cell-autonomous manner and increased by osteoclast-targeted transgenic ATF4 expression. ATF4 assists osteoclast differentiation by directly activating the gene encoding the critical transcription factor NFATc1, or modulating RANKL activation of multiple MAPK pathways in osteoclast progenitors, or mediating M-CSF induction of RANK expression on bone marrow monocytes (BMM), a critical step for early osteoclast differentiation. The lack of ATF4 causes a shift in osteoclast precursor to macrophage, thereby increasing macrophage. Furthermore, the level of ATF4 protein is largely modulated by M-CSF signaling and the PI3K/AKT pathways in BMM. Finally, ATF4 is upregulated by myeloma cells-derived factors such as TNFa in primary mouse BMM cultures. These results demonstrate that ATF4 plays a direct osteoclast-intrinsic role in regulating osteoclast differentiation and suggest that it may be a therapeutic target for treating bone diseases associated with increased osteoclast activity.

Bidirectional interactions between cancer cells, immune cells, and stromal cells were an important topic at the conference. Dr. Jeffery Pollard from Albert Einstein Medical College, USA, talked about macrophages promoting tumor progression and metastasis. He pointed out that there is persuasive clinical and experimental evidence that macrophages promote cancer initiation and malignant progression. Macrophages enhance malignancy at the primary site by stimulating angiogenesis, inducing tumor cell migration, invasion and intravasation and by suppressing anti-tumor immunity. At metastatic sites macrophages promote tumor cell extravasation, survival and subsequent growth. Each of these activities is stimulated by a different population of macrophages whose unique signaling pathways might represent new therapeutic targets. Dr. Limin Zheng from Sun Yat Sen University, China, also spoke about activated macrophages in human tumors. He showed that tumor environment can alter the normal development of macrophages that is intended to trigger transient early activation of monocytes in the peritumoral region. Dr. Zheng pointed out that it is not inflammation alone but rather the inflammatory "context" that determines the ability of pro-inflammatory factors to facilitate or prevent tumor growth.

Dr. Wolf-Herve Fridman, Cordeliers Research Centre, INSERM, University Paris Descartes and University Pierre et Marie Curie, France, also spoke about immune control of human tumors. He emphasized that the microenvironment of human tumors differs from patient to patient in their lymphocytic infiltration. In addition, lymphocytes are not randomly scattered within the tumor but appear organized in the center of the tumor, its invasive margin and in tertiary lymphoid follicles adjacent to the tumor nests. His lab has particularly analyzed large cohorts of colorectal and lung cancers and have shown that a high infiltration of memory $T$ cells, with a Th1 and cytotoxic orientation, both in the center and the invasive margin of the primary tumors is a major prognostic factor since it controls tumor recurrence, probably through an immune control of potential metastatic cells. He and colleagues recently analyzed some of the factors essential to shape an efficient immune control, which is likely to originate in the tertiary lymphoid follicles, and identified chemokines and cytokines produced in the microenvironment which structures a T cell specific response. These data allow drawing an integrated picture of how an immune reaction develops in the tumor microenvironment to control cancer spread and recurrence.

Dr. Michael Lotze, University of Pittsburgh School of Medicine, discussed damage associated molecular pattern molecules that drive altered tumor metabolism and inflammation. His lab's recent discovery of cytosolic HMGB1 as an inducer of autophagy and others of cytosolic p53 as an inhibitor of autophagy has placed these two molecules at the crux of metabolism. He pointed out a novel role for HMGB1 as a cytosolic factor promoting autophagy and mitophagy, enhancing aerobic glycolysis, rapid ATP generation, and limiting apoptosis, and concluded that HMGB1 serves as a critical link, regulating metabolism, the response to stress, and cellular survival.

Dr. Zhihai Qin, Institute of Biophysics, Chinese Academy of Sciences, China, discussed the role of fibroblast in tumor microenvironment. Stromal fibroblasts can not only synthesize extra-cellular matrix, which is crucial for the encapsulation of the tumor, but also secrets a variety of cytokines regulating tumor angiogenesis, recruitment and activation of different immune cells. He showed that fibroblast-involved "foreign body reaction" against tumor cells is inhibitory to carcinogenesis. Using a model by coinjection of tumor cells and gene-modified mouse embryonic fibroblasts, he demonstrated that fibroblast inhibits tumor growth via IFNY-mediated down-regulation of VEGF expression by these cells.

Other factors in tumor microenvironment, such as fatty acids, were also discussed. Dr. Yifan Dai from Nankai University spoke about the tumor inhibitory effect of omega-3 fatty acids, an essential fatty acid that has to be taken from diet. An unhealthy ratio of omega-6/-3 may be attributed to many modern human diseases such as obesity, diabetes, coronary heart diseases, and cancer. By using transgenic mice in which omega- 6 is converted to omega- 3 
fatty acids by the enzyme Fat-1, he showed that omega-3 fatty acids have significant inhibitory effect on hepatocellular carcinoma, colon cancer, prostate cancer and breast cancer.

The conference was hosted by Nankai University College of Pharmacy and The National Basic Research Program of China (973 Project) on Cancer Microenvironment at Nankai, headed by Dr. Lu-Yuan Li. The audience consisted of medical researchers and graduate students from universities and research institutions in China. Many felt that the conference served its intended purpose: to help establish a tumor microenvironment research field in China.

\section{ACKNOWLEDGEMENTS}

The conference was supported in part by grants from The Ministry of Science and Technology of China (Grant No. 2009CB918900), Tianjin Heng Xing Cheng, Inc., Beijing Sunjoy, Inc., Eppendorf Shanghai, and Millipore Corporation. 